\title{
DEMORA EN TIEMPOS
}

DE RESPUESTAS A

CODIGOS AMARILLOS

EN UN SERVICIO DE EMERGENCIAS MÉDICAS DE LA CIUDAD DE CORDOBA

DELAY IN ANSWERING YELLOW CODES IN A MEDICAL EMERGENCY SERVICE IN THE CITY OF CORDOBA

DEMORA NOS TEMPOS DE RESPOSTA AO CÓDIGO AMARELO EM UM SERVIÇO DE EMERGÊNCIA MÉDICA NA CIDADE DE CÓR$D O B A$

Alvarez Ricardo E. 1 Andrómaco Marta I. ${ }^{2}$

${ }^{1}$ Médico Especialista Terapia Intensiva y

Emergentología. UTI 2 -Hospital San Roque Magister en Gerencia y Administración en Servicios de Salud ${ }^{2}$ Medica

Especialista en Educación Médica y Profesor Consulto

Universidad Nacional de Córdoba

Resumen de tesis de Maestría de gerencia y Administración de servicios de salud. Escuela de Salud Pública FCM UNC

Trabajo recibido: 13 de mayo de 2016.

Aprobado: 16 de septiembre de 2016.

\section{Resumen}

Este estudio trata las causas de demora en los tiempos de respuestas a códigos amarillos en un servicio privado de emergencias médicas de la ciudad de Córdoba. Los niveles de prestaciones son diferenciados por códigos según riesgo de vida y/o por tiempo de respuesta en: Verdes (consultas o demora hasta 3 horas), Amarillos (urgencias o demora hasta 20 minutos), Rojos (emergencias o demora hasta 12 minutos) Azul (Traslados, sin demora establecida). Como no se cumplen los convenios realizados por la empresa en Tiempo de Respuesta al código amarillo, se analizan las etapas del proceso, se observan sala de despacho y móviles operativos, se realizan encuestas y entrevistas a los actores claves.El tiempo de Respuesta (TR) promedio total fue de 42:26 minutos, los TR fuera de zona de influencia del móvil (FZ) 50:23, si tomamos T.R. en horario de picos de llamadas, de 09 a 14 hs. este fue de 37:42 minutos, en el mismo horario los T.R. FZ fue de 44:55 minutos, en horario pico de 18 a 22 hs T.R. encontrado 46:36 minutos 
y F.Z. 53:27 minutos. Se encuentra demora en el despacho de 12,32 minutos y la salida de móvil de 6,20 minutos consumiendo entre ambos el 43\% del Tiempo de Respuesta total. La mayor demora ocurre durante los horarios picos de llamadas que tiene una curva bimodal en el transcurso del día, acentuando esa demora cuando son asignados a móviles fuera de su zona de influencia. La demora en el despacho se atribuye a la falta de disponibilidad de móviles, se asignan códigos verdes y amarillos comerciales para que el móvil cumpla con productividad lo que genera menor capacidad de respuesta al tener los móviles ocupados. La demora en salida del móvil se debe al malestar del personal operativo que además no toma el tiempo de respuesta como prioridad. No se evidenció una adaptación de la oferta operativa a la mayor demanda de códigos Amarillos por convenios.

Palabras clave: Urgencia,Tiempo de respuesta, código amarillo, capacidad respuesta, Sistema Prehospitalario.

\section{Abstract}

This study deals with the causes of delay in answering yellow codes in a private service of medical emergencies in the city of Cordoba. The levels of services are differentiated by codes according to risk of life and/or answering time in: Green (consultation or delay up to 3 hours), Yellow (urgencies or delay up to 20 minutes), Red (emergencies or delay up to 12 minutes), Blue (transfer of patients, no delay established). As the company agreements are not observed regarding answering time to yellow code, the stages of the process are analyzed, dispatching room and operative vehicles are observed and surveys and interviews to key participants are carried out. Total average Answering Time (AT) was 42:26 minutes; AT outside the area of influence of the vehicle (OA) was 50:23. If we consider AT in incoming call pick hour from 9am to $2 \mathrm{pm}$, it was 37:42 minutes; in the same timetable AT OA was 44:55 minutes. In peak hour from $6 \mathrm{pm}$ to $10 \mathrm{pm}$ AT found was $46: 36$ and OA 53:27 minutes. A 12.32 minutes delay was found in dispatching and 6.20 minutes in vehicle departure taking up $43 \%$ of Total Answering Time. The largest delay takes place during incoming call peak hours having a bimodal curve during the day; this delay is emphasized when vehicles are assigned outside the influence area. Delay in dispatch is put down to lack of availability of vehicles, commercial green and yellow codes are assigned so that the vehicle complies with productivity generating less answering capacity because vehicles are busy. Delay in vehicle departure is due to discomfort of operative staff who besides, does not take answering time as a priority. Adaptation of operative offer to larger demand of yellow codes by agreements was not shown.

Key words: Urgency, Answering Time, Yellow Code, Answering Capacity, Prehospital System.

\section{Resumo}

Este estudo trata das causas de atraso no tempo de resposta aos códigos amarelos em um serviço privativo de emergência médica na cidade de Córdoba. Os níveis de desempenho são diferenciados por códigos segundo o risco de vida e / ou tempo de resposta: Verde (consulta ou demora de até 3 horas), Amarelo (emergencia ou demora de até 20 minutos), Vermelho (emergencia ou demora de até 12 minutos), Azul (Traslados, sem demora estabelecida). Sendo que os acordos feitos pela empresa para os Tempos de Resposta ao código amarelo não foram cumpridos, as etapas do proceso são analisadas, telefones de escritório e sala de operação são observados, enquetes e entrevistas são conduzidas com os atores- chave. O tempo de Resposta (TR) médio total foi de 42:26 minutos, os TR fora zona de influência 
do veículo de transporte (FZ) 50:23, se considerarmos o TR em horas de pico dos requerimentos, de 09 a 14 horas. Este foi de 37:42 minutos, no mesmo horário, o T.R. FZ foi de 44:55 minutos, no horário de pico de 18 a 22 hs o T. R. achado foi de 46:36 minutos e o F.Z. de 53:27 minutos. Acha-se demora no despacho de 12,32 minutos e na saída do veículo de 6,20 minutos que consomem entre ambos os dois um $43 \%$ do Tempo de Resposta Total. A maior demora ocorre durante horários de pico de requerimentos que apresenta uma curva bimodal no curso do dia, enfatizando-se o dito atraso no caso de veículos fora da sua área de influência. $\mathrm{O}$ atraso no despacho é atribuído à falta de disponibilidade de veículos, códigos verdes e amarelos comerciais são atribuídos para o veículo cumprir com sua produtividade o qual gera menor capacidade de resposta por causa de os móveis ficarem ocupados. A demora na saída do veículo é devida ao desconforto do pessoal de operação por desconsiderar o tempo para responder como sendo uma prioridade. Não foi evidenciada uma adaptação da oferta operacional para o aumento da procura de códigos Amarelos por convênios.

Palavras-chave: Urgência, Tempo de resposta de emergência, código amarelo, capacidade, resposta, Sistema Pré-hospitalar.

\section{Introducción}

Cuando hablamos de calidad en servicios de salud, frecuentemente los médicos la relacionamos solo con calidad asistencial científico-técnica, pero debemos tener en cuenta que tan importante es la percepción del usuario, tanto en el tiempo de respuesta como en el trato personalizado, elementos básicos de calidad percibida. Estos indicadores de calidad son indispensables para conocer la satisfacción del usuario y la continua mejora del servicio.

El Tiempo de Respuesta en un servicio de emergencia es un indicador de proceso de servicio que además de ser un indicador de calidad junto a la percepción de satisfacción del usuario por la calidad asistencial; sirve para la toma de decisiones globales del servicio, permite además conocer la agilidad de cada equipo de trabajo, el valor individual y grupal de productividad y orienta a estrategias logísticas de ubicación de bases operativas, cantidad de móviles en cada período del día, del mes y del año.

La adaptación de la oferta a la demanda constituye un elemento indispensable en la gestión de servicio. Las políticas de las empresas privadas fueron mutando con los años y adecuándose a las exigencias del mercado, que cada vez más se inclina a obtener tiempos de respuesta menores a costo de perder la calidad asistencial, tanto por abaratar costos, como por brindar más carga de trabajo a la misma cantidad de recursos humanos y que el tiempo de asistencia sea menor; aun así, se observa con preocupación que no pueden cumplirse.

Concepto de URGENCIA - OMS y Organización Panamericana de la salud: "Situación clínica con capacidad para generar deterioro o peligro para la salud o la vida de un paciente en función del tiempo transcurrido entre su aparición y la instauración de un tratamiento efectivo, que condiciona un episodio asistencial con importantes necesidades de intervención, en un corto periodo de tiempo".

Concepto de COMPLEJIDAD - OMS y Organización Panamericana de la salud: Cualidad relacionada con el grado de dificultad diagnóstica y/o terapéutica debida a la presencia de complicaciones y/o co-morbididades (diagnósticos secundarios) añadidas al diagnóstico principal que nos permite prever un tiempo de estancia y/o un coste determinado.

Respecto a la definición de URGENCIAS:"No hay urgencias, sólo hay gente apurada". Criterio restrictivo

"Todo lo que se siente como urgente es urgente" Criterio Laxo

El factor Tiempo $(\mathrm{T})$ Ya dijimos mientras menor es T, mayor es la Urgencia $(1 / \mathrm{T})$. 
MartínezAlmoyna Propone que el Quantum de Urgencia es igual a

$$
\mathrm{U}=\mathrm{G} \times \mathrm{T} \times \mathrm{C} \times \mathrm{V}
$$

G: grado de gravedad. T: tiempo, C: grado de cuidado y V: valencia social.

El Dr Miguel Martinez Almoyna (SAMU de Paris) en su manual de regulación médica de los SAMU da una definición abierta de URGENCIA para analizar y razonar: "ES UN CONJUNTO DIFUSO DE CONJUNTOS DIFUSOS".

Un paciente declarado urgente tiene un status prioritario por sobre los demás pacientes, se le otorga esto por la razón de que puede evolucionar a un estado más grave al punto que no puede permanecer en la cola de espera, esto plantea un tratamiento enérgico con procedimientos expeditivos con conductas de menor calidad y asumiendo riesgos anormales que a menudo puede terminar en una atención de menor calidad. Este estado prioritario puede también ser definido como Valencia Social (V). Desde el punto de vista estrictamente médico también es confuso definirla ya que muchas veces sufre variaciones aún en el propio médico según su posición, que a veces excluyen de esta clasificación a otros pacientes que sufren la misma patología.

\section{Objetivos}

Objetivo general: Identificar causas de demoras en los tiempos de respuestas de los códigos amarillos realizando un análisis exhaustivo de los procesos, actores, características propias de la empresa y su entorno.

Objetivos específicos:

$>$ Describir las Fases del proceso de tiempos de respuesta a códigos Amarillos.

$>$ Identificar y comparar tiempos en cada fase del proceso.

$>$ Conocer que genera la demora en los tiempos de respuesta a códigos Amarillos para tratar de encontrar sugerencias que permitan mejorar el tiempo de respuesta hacia los mismos.

> Proponer a partir de este análisis y las conclusiones obtenidas algunas recomendaciones para mejora de los tiempos de respuesta.

\section{Metodologia}

Estudio descriptivo, retrospectivo, longitudinal. Se utilizó método de gestión por procesos, se construye un Mapa de Procesos con Flujograma, donde se analizan las diferencias de tiempo en cada fase de los procesos operativos que componen los tiempos de respuesta a los códigos Amarillos.

Se realiza observación presencial del Centro de Despacho Informático CEDI, con encuestas vinculadas a informantes claves como Gerente Médico, jefe de Área Encargada de área de Convenios y Marketing utilizando escala de Likert con preguntas abiertas.

La selección de muestra: del universo de llamados ocurridos en la empresa durante el período que va del 01 de enero al 30 de junio de 2014, se obtuvo un subgrupo que corresponde al fenómeno en estudio( los códigos asignados inicialmente con el color amarillo que son los que generan el problema de demora en tiempo de respuesta.

Recolección de datos: Se obtiene de una planilla Excel que la empresa recaba de manera automática con los datos que se van cargando.

Código Rojo (Emergencia) de mayor prioridad con un tiempo de llegada teórica dentro de los 12 minutos de recepción de llamada.

Código Amarillo (Urgencia) de segunda prioridad con un tiempo teórico de llegada dentro de los 20 minutos.

Código Priorizado (Urgencia menor) tercera prioridad, tiempo teórico de llegada 40 minutos.

Código Verde (Consulta) cuarta prioridad, tiempo teórico de llegada 120 minutos. 


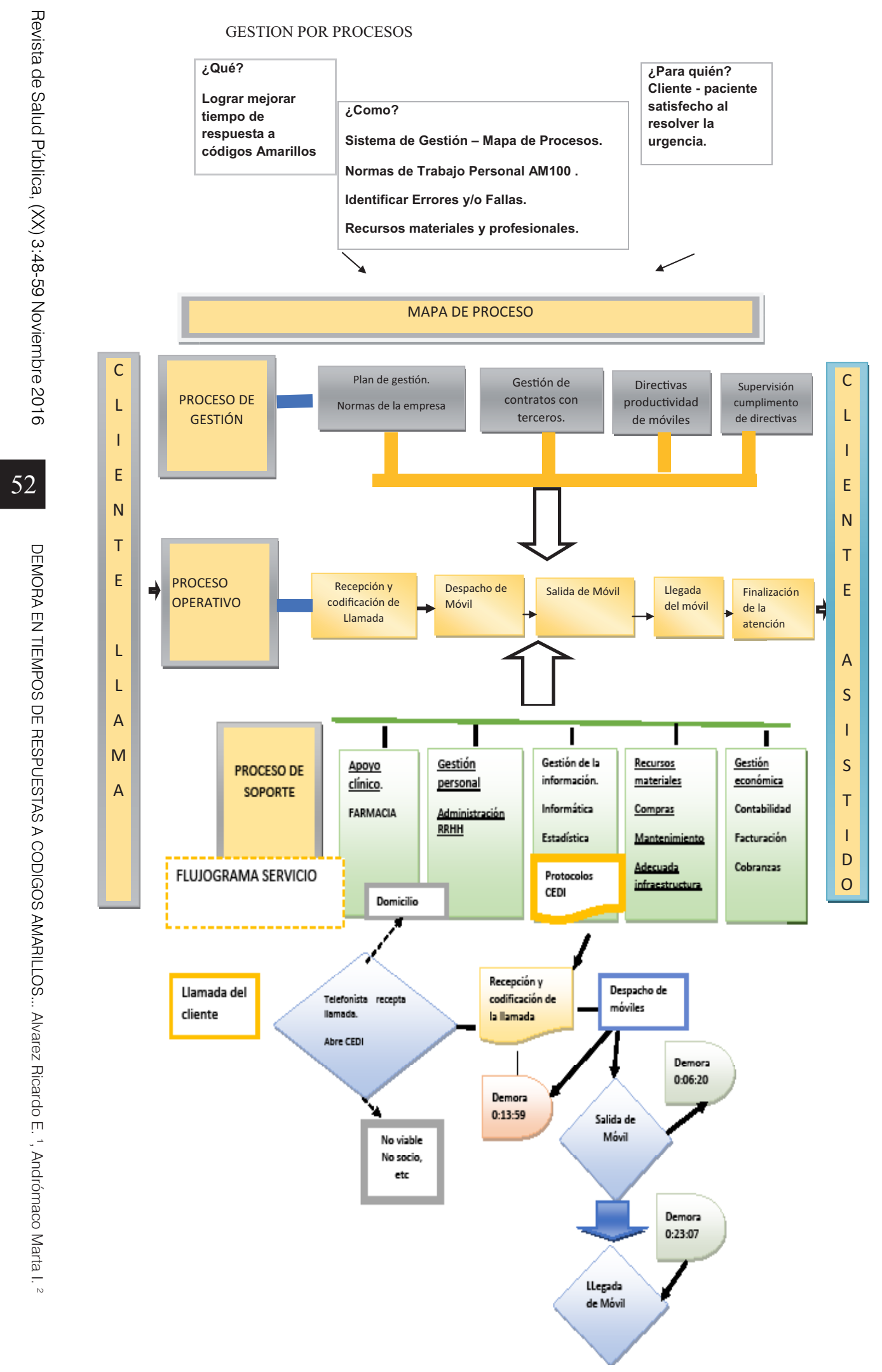




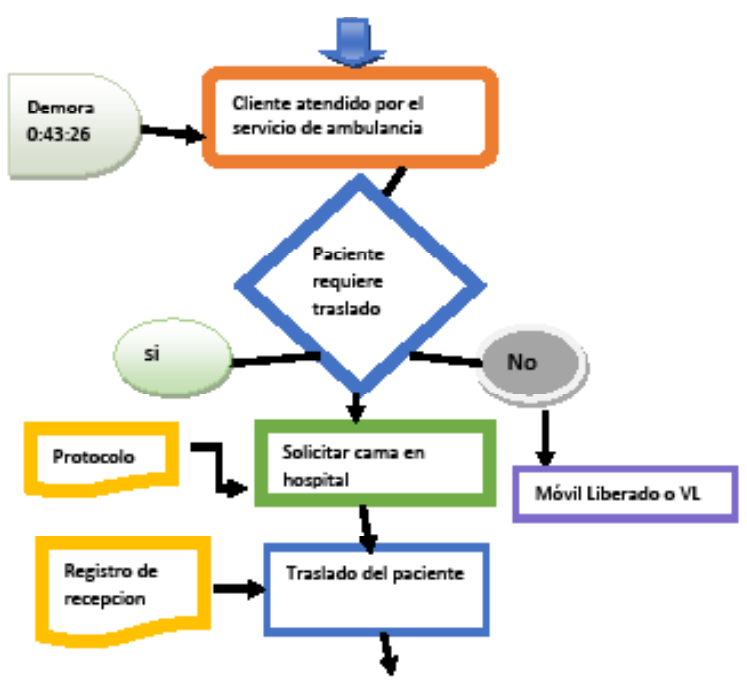

Resultados

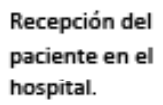

Recepción del

paciente en el

hospital.

A continuación se presentan los mismos en tablas y graficos.

Tabla 1 Tiempos de cada proceso totales y en Códigos Amarillos fuera de zona

\begin{tabular}{|l|l|l|l|l|l|l|}
\hline Tiempo/Proceso & Recepción & Asignación & $\begin{array}{c}\text { Salida } \\
\text { Móvil }\end{array}$ & $\begin{array}{c}\text { Llegada } \\
\text { Móvil }\end{array}$ & $\begin{array}{c}\text { Atención } \\
\text { sin } \\
\text { derivación }\end{array}$ & $\begin{array}{c}\text { Resolución } \\
\text { /derivación } \\
\text { total }\end{array}$ \\
\hline Total & $0: 1: 27$ & $0: 12: 32$ & $0: 06: 20$ & $0: 23: 07$ & $0: 32: 14$ & $1: 40: 38$ \\
\hline $\begin{array}{l}\text { Fuera de } \\
\text { Zona }\end{array}$ & $0: 1: 32$ & $0: 13: 10$ & $0: 10: 18$ & $0: 25: 22$ & $0: 32: 16$ & $2: 10: 43$ \\
\hline
\end{tabular}

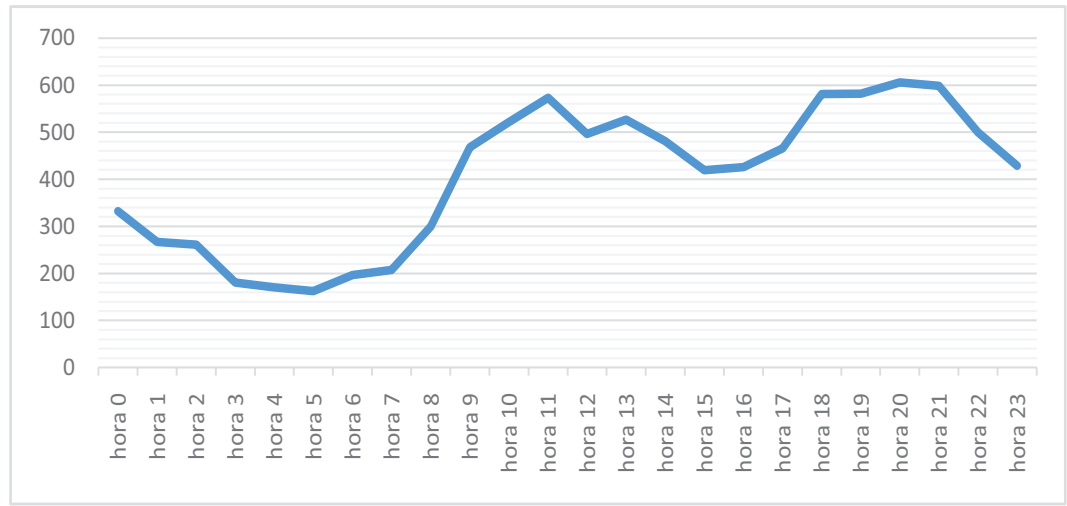

Figura 1: Hora de Curso

Hora de curso, se observa curva bimodal con dos horarios picos uno de 09 a 14 hs. y otro de 18 a 22 hs 


\section{1,27 \\ $3 \%$}

23,07

$54 \%$
- Tiempos/procesos Total TR Recepcion

12,32

$29 \%$
- Tiempos/procesos Total TR Despacho

- Tiempos/procesos Total TR Salida Móvil

- Tiempos/procesos Total TR Trayecto Móvil

Figura 2: tiempos/proceso total.

Analizando los tiempos del proceso vemos que el 28,4\% lo ocupa la demora en asignación de móvil y el 14,3 \% la demora en salida de móvil, sumando un $43 \%$ entre ambos

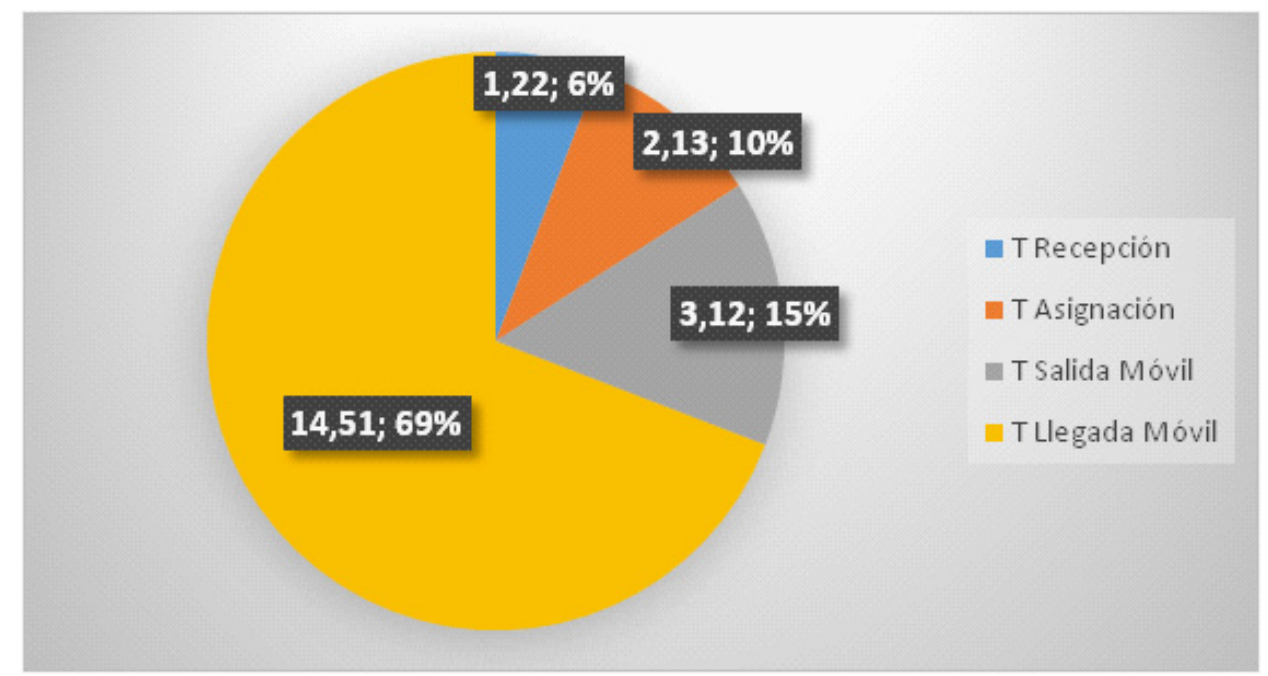

Figura 3: tiempos/ proceso TR

En códigos Amarillos que cumplen con T.R de hasta 20 minutos se observa que la demora en Asignación es 15\% y la salida de móvil del 10\%, el trayecto aumenta al 69\%, pero disminuye en tiempo real 


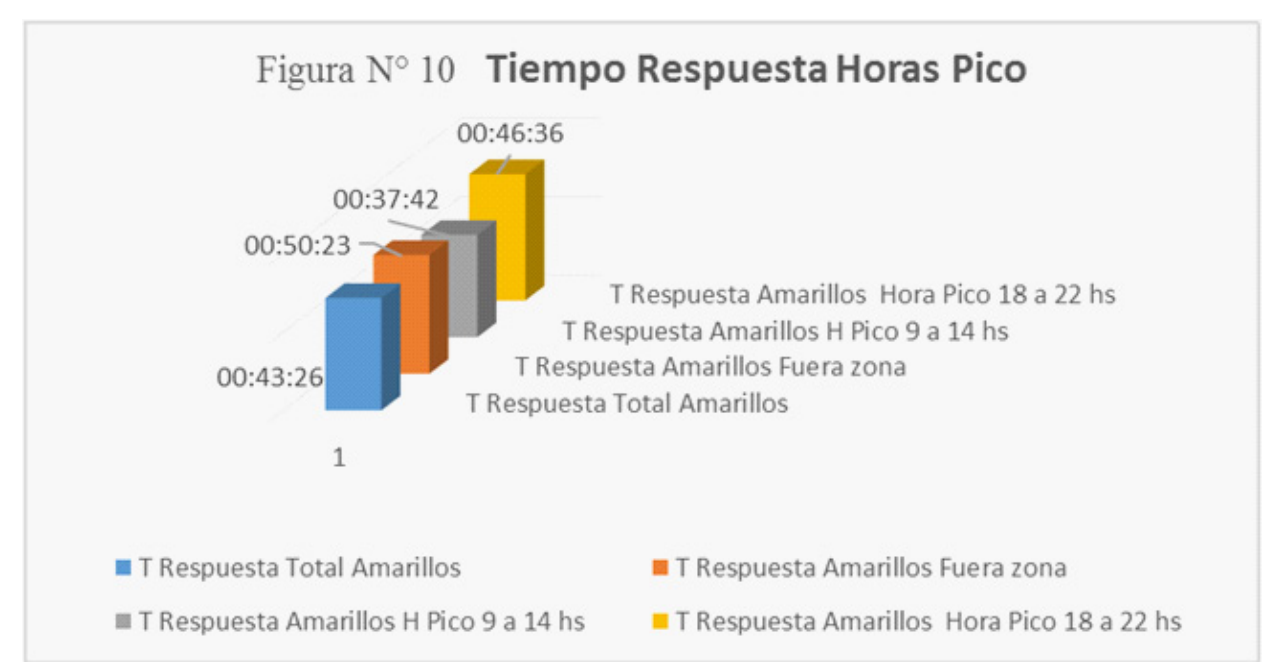

Figura 4: Tiempo respuesta horas pico

Se evidencia un significativo aumento del Tiempo de Respuesta cuando son fuera de zona y se acentúa en horarios picos franja horaria de 18 a 22 hs.

\section{Discusión}

Tiempo de Respuesta es un indicador de calidad utilizado por los Servicios de Emergencias Médicas junto a la calidad asistencial, este proceso se evidencia en la bibliografía revisada, cuyos resultados son utilizados como herramienta para mejorar la gestión y como garantía de satisfacción para usuarios y el sistema en general, tal como lo explica el servicio Andaluz de salud (24).

Comparando los tiempos encontrados en este estudio con el revisado, se observa una gran diferencia en los procesos de tiempo de despacho y en tiempo de salida de móvil (1), (6), (8), (13), el primero en la mayoría de la bibliografía no debe ser mayor a 1 minuto, el segundo en algunos estudios lo dan como valor cero (5), (6), (17), excepcionalmente no debe superar un minuto, los tiempos promedios encontrados en este estudio son 12:32 minutos y 6:20 minutos respectivamente, siendo la suma de estos dos tiempos el $43 \%$ del total del proceso.

Investigando bibliografía encuentro que no existen normas locales, provinciales, nacionales ni internacionales que sean claras y específicas, los trabajos se rigen por normas propias de cada sistema público o empresa privada (1), (11), (20), (23).

\section{Conclusiones}

El tiempo de Respuesta es el tiempo que transcurre desde que el afiliado accede al sistema hasta que el personal operativo toma contacto con él mismo, esto es un proceso cuya primera fase inicia con la recepción del llamado, calificación de código Amarillo, continúa con la aparición de los datos en la pantalla del despachante quien selecciona y decide según el móvil libre que tenga más cercano al lugar, asignando dicho código, continúa cuando el personal operativo toma conocimiento y procede a salir de base para dirigirse al domicilio asignado, extendiendo la última fase al trayecto del móvil hasta tomar contacto con el afiliado, allí finalizan las fases del proceso de Tiempo de Respuesta.

Las mayores demoras encontradas ocurren en las fases de despacho de móvil y en la de salida de móvil que consumen casi la mitad del total de Tiempo de Respuesta, siendo estos valores excesivos para las normas fijadas. 
Se encuentra demora en la fase de despacho fundamentalmente en los horarios picos de la curva bimodal, esto es debido a falla en la capacidad de respuesta traducida en falta de disponibilidad de móvil libre en la zona.

Basado en las encuestas y observación presencial, la demora en la salida de móvil se atribuye al perfil asistencialista del personal operativo, que prioriza una atención de calidad por sobre cumplir con el tiempo de respuesta.

En cuanto al aspecto operativo contar con la capacitación correspondiente, además del generar compromiso y trabajo en equipo entre personal de despacho y personal de móviles, todo esto evaluado con monitoreo continuo.

Reorganizar la ubicación y número de bases operativas acordes al crecimiento de la Ciudad de Córdoba.

\section{Bibliografía}

1) Carrieri F. A., Marinucci J., Navarret N., Otero Vidal L., Rois O. 1993. Manual de despacho de los Sistemas de Emergencias Médicas Prehospitalarias. Primera Edición - Uruguay 1993.

2) Paredes Z.G. 2004. Indicadores de Emergencia Prehspitalaria. La Calidad en Medicina de Emergencia UGRAV 2004 - Bogotá - Colombia.

3) Andersen M. Set col. 2013. Implementein a Nationwide criteria-based emergency medical dispatch system: A register based follow-up study. Scandinavian Journal of Trauma, Resuscitation and Emergency Medicine 2013, 21:53.

4) Martinez Almoyana, Miguel. Manual de Regulación Médica de los SAMU : Elementos para la Regulación Médica de las Urgenticas. Buenos Aires : SAMU, 1998. Disponible en:

http://www.buenosaires.gob.ar/areas/salud/same/publicaciones/man_reg_ medica_1.pdf

5) Eisemberg M.S. 1979 Cardiac Resuscitation in the Community importance of rapid provision and implications for program planning. Jama 1979; 241: 1905-1907.

6) Blackwell T.H et. Al. 2002.Response Time Effectiveness: Comparison of Response Time and Survival in an Urban Emergency Medical Services System. AcadEmerg Med 2002;9:288-295.

7) Gómez J. Jimenez, M. R. y col 2008. Triaje Estructurado en los Servicios de Urgencias. Documento de Posición de la Societat Catalana de Medicina d'Urgències i Emergències (SCMU-ACMES). Documentaprovat per la Junta directiva de SCMU-ACMES el 31 de gener de 2008.

8) Ong, Eng Hock Marcus. REDUCCIÓN DE LOS TIEMPOS DE RESPUESTADE LA AMBULANCIA USO GEOSPATIAL-TIEMPO ANÁLISIS DE AMBULANCIA DESPLIEGUE. Singapur : Emergencia Medicina Académica. 2010

9) Raven M.C et col. 2013. Comparison of Presenting Complaint vs Discharge Diagnosis for Identifying "Nonemergency" Emergency Department Visits. JAMA, March 20, 2013-Vol. 309, No. 111145.

10) Sistema de Atención pre hospitalaria. Gestión de emergencias Médicas. [en Línea]. Argentina. Ley 1.883. BOCBA; 2006. [ 13/01/2015]. Disponible en: http://www2.cedom.gob.ar/es/legislacion/normas/leyes/ley1883.html

11) Perú. Ministerio de Salud. Norma técnica de los servicios de emergencia de hospitales del sector salud. Dirección General de Salud de las personas. 2004 Disponible en:

http://www.minsa.gob.pe/portalminsa/destacados/archivos/44/Norma\%20 T\%C3\%A9cnica\%20Emergencias.pdf

12) V F Turner, VF; Bentley, PJ; Hodgson, SA; Collard, PJ; Drimatis, R.; Rabune, C.; Wilson, AJ. Telephone triage in Western Australia. MJA 2002; 176: 100-103 
13) Turner J. 2011 Should Response Times continue to be used for Performance Measurement and Targets?Prehosp Disaster Med 2011;26(Suppl. 1):s23 doi:10.1017/S1049023X11000823. Abstracts - 17th World Congress on Disaster and Emergency Medicine

14) Henrik Jaldell a, Dr. Lebnak P b, Dr. Anurak A 2013 Time is money, but how much? Karlstad University Working Paper in Economics \# 2013 / 2

15) Vargas Román, MI; Gil de Miguel, A.; Carrasco Garrido, P.; Suárez Bustamante, R.; Medina Álvarez, JC.; Gilarranz Vaquero, JL. GRAVEDAD Y SUPERVIVENCIA DE PACIENTES ATENDIDOS POR UN SERVICIO DE EMERGENCIA SANITARIA PREHOSPITALARIA. Emergencias 2005; 17:44-51

16) Argentina. Ministerio de Salud. Instructivo de recomendaciones básicas médicolegales: para situaciones de emergencia. 2010. Disponible en:

http://www.msal.gob.ar/dinesa/images/stories/pdf/rec_med_leg.pdf

17) Fischer, Matthias. Factors Influencing Outcome After Prehospital Emergencies. Germany: Department of Anaesthesiology and Intensive Care Medicine, 2007. Disponible en:

http://www.esahq.org/ /media/ESA/Files/Refresher\%20Courses/2007/Factors\%20 influencing\%20outcome \%20after\%20prehospital\%20emergencies\%20\%20A\%20 European\%20perspective\%20(2007).ashx

18) Matthew Gratton,, MD; Garza, Alex.; Salomone, Joseph A.; McElroy, James; Shearer, Jason. Clasificacion de las ambulancias Respecto a los escenarios pontencialemente peligrosos: otro componente oculto del tiempo de respuesta. PREHOSPITAL EMERGENCY CARE. 2010 ; 3( 4) p. 201-207. Disponible en: file://C:/Users/Biblioteca1/Downloads/X1888402410903285_S300_es.pdf

19) Perú. Ministerio de Salud. Comisión Multisectorial. Informe técnico final de la comisión multisectorial encargada de proponer los mecanismos que permitan consolidar un Sistema Nacional de salud. R.M. No 866- 2008-SA. Disponible en: ftp://ftp2.minsa.gob.pe/descargas/Profesionales/sns/Archivos/ITCM InformeFinal01-13.pdf

20) Ambulancias aún no cumplen el tiempo óptimo de respuesta. La Hora Nacional. Miércoles 14 de agosto 2013., Reporte. Disponible en:

http://lahora.com.ec/index.php/noticias/show/1101548674/-1/ Ambulancias_a\%C3\%BAn_no_cumplen_el_tiempo_\%C3\%B3ptimo_de_ respuesta.html\#.V9vizPB97cc

21) Victoria Prado, Carlos Antonio. Situación Actual de la Atención de Urgencias Médicas en la ciudad de México. México. 2004. Disponible en:

http://www.disaster-info.net/lideres/spanish/mexico2004/presenta/Alumnos/ CarlosVictoria/Situacion.pdf

22) Organización Panamericana de la Salud. Gestión de la información y comunicación en emergencias y desastres: guía para equipos de respuesta. Washington: OPS/ OMS. 2009. Disponible en:

http://www.msal.gob.ar/images/stories/ryc/graficos/0000000749cnt-2014-04_gestioninfo-comunicacion-desastres.pdf

23) Tejedor Fernández, Martín, coord. Manual de indicadores de actividad y calidad para urgencias y emergencias sanitarias. Andalucia: Servicios de Andaluz de Salud. Junta de Andalucia Conserjería de Salud; 2000. Disponible en: http://www.medynet. com/usuarios/jraguilar/Manual\%20Indicadores\%20Calidad.pdf

24) . Dirección General de Emergencias y Protección Civil .SAMUR - Protección Civil. Memorias 2010 -2011. Ciudad de Madrid. España

25) Dirección General de Emergencias y Protección Civil. SAMUR - Protección Civil. Memorias 2012 -2013. Ciudad de Madrid. España.

26) Organización Panamericana de la Salud. Gestión de la información y comunicación 
en emergencias y desastres: guía para equipos de respuesta. Washington: OPS/ OMS. 2009. Disponible en:

http://www.msal.gob.ar/images/stories/ryc/graficos/0000000749cnt-2014-04_gestioninfo-comunicacion-desastres.pdf

27) Sabriá, Federico. Estudio de Sistemas de respuesta a Emergencias en Carreteras y Autopistas (SIRECA) 2010

28) Hancok, abkowitz y Lepofsky. Capacidad de respuesta a Eemrgencia. 1992. Disponible en:

http://www.eird.org/cdfororegional/pdf/spa/doc15774/doc15774-4.pdfSistema de Atención pre hospitalaria. Gestión de emergencias Médicas. [en Línea]. Argentina. Ley 2127. BOCBA; 2006. [1/12/2006]. Disponible en: http://www2.cedom.gob.ar/ es/legislacion/normas/leyes/ley2127.html

29) Bogotá. Sistema distrital de Prevención y Atención de Emergencias.Guía para la elaboración de planes nstitucionales de respuestas a emergencias por Terremotos. 2002. Disponible en: http://www.ridsso.com/documentos/muro/db222dbed4d03ad e4a205b0680c047a6.pdf

30) Valdivia López, Armando. LOS TIEMPOS DEL SISTEMA DE SERVICIOS MEDICOS DE EMERGENCIA (SSME). Octubre 2009

31) Peter T. Pons, MD, Jason S. Haukoos, MD, MS, Whitney Bludworth, MD, Thomas Cribley, EMT-P, Kathryn A. Pons, RN, Vincent J. Markovchick, MD. Paramedic RESPONSE TIME: DOES IT AFFECT PATIENT SURVIVAL? Pons et al. d Paramedic response time. ACADEMIC EMERGENCY MEDICINE 2005; 12:594600

32) Lira Villavicencio, Manuel. Tiempo de respuesta de los transporte de prioridad I y II en el servicio de STAE. Portales Médico.com. 2007. Disponible en: http://www.portalesmedicos.com/publicaciones/articles/439/1/Tiempo-derespuesta-de-los-transportes-primarios-de-prioridad-I-y-II-en-el-servicio-de-stae-essalud.html

33) Pinet, Luis M.. Atención prehospitalaria de urgencias en el Distrito Federal: las oportunidades del sistema de saludSalud Pública de México [en linea] 2005, 47 (enero-febrero) : [Fecha de consulta: 6 de septiembre de 2015] Disponible en: $<$ http://www.redalyc.org/articulo.oa?id=10647110> ISSN 0036-3634

34) Kathleen M. Brown, MD, Charles G. Macias, MD, MPH, Peter S. Dayan, MD, MSc, Manish I. Shah, MD, Tasmeen S. Weik, DrPh, MPH, Joseph L. Wright, MD, MPH, Eddy S. Lang, MDCM, CCFP (EM) PrehospEmerg Care Downloaded from informahealthcare.com by 186.138 .39 .141 on 10/06/14 THE DEVELOPMENT OF EVIDENCE-BASED PREHOSPITAL GUIDELINES USING A GRADE-BASED METHODOLOGY

35) Sánchez Losada, JA., Romero Sánchez, S., Romero Sánchez, R., Pavón Olid, J. Toma de decisiones en urgencias sanitarias: regulación médica. Emergencias y Catástrofes. 2001; 2(4): 197-201 